\title{
La fórmula de comunicación de L asswell como método para implementar bases de datos documentales en los medios audiovisuales
}

\author{
Jorge Caldera Serrano \\ Facul taddeBiblio teconomíay Documenta ción, Universidadde \\ Extremadura,EdificioAlcazaba, s/n.06071 Badajoz, \\ Es pa ña, + 34924286400 Ext. 6440 \\ E-mail:jcalser@alcazaba.unex.es \\ FELIPE Zapico Alonso \\ FacultaddeBiblioteconomíayDocumentación, Universidadde \\ Extremadura, Edificio Alcazaba, s/n,06071 Ba dajoz, \\ Es pa ña, + 34924286499 Ext. 6422 \\ E-mail:fzapalo@alcazaba.unex.es
}

\section{RESUMEN}

Elmo delo de co munica ción deLass welles el méto do acepta do porla comunidad de periodistas como fórmula de relación comunicativa con el teles pectador. Plantealanece sidadde que elmen sajepe riodís tico re suel va una se rie de cues tio nes ta les como el qué, cómo, cuán do, dón de y por qué. Este mo delo ha de riva do en las de no mina das reglas delas cin co W, que incluyen los elementos comunicativos que deben evidenciarse en cualquier proce so en tre emisory re cep tor. El méto do es tambiénacep tado para los me dios de co municación au dio visua les porlo que pa re ceun modeloválidoparaimplementarlas bases de datos documentales. Así, si lapieza au dio visualin forma tiva se con feccionacontestandolaspreguntas planteadas en el modelo sería oportunodesmembrar dichainformación nuevamente, y conservarla y almacenarla en las bases de datos por medio de campos específicos.

Pa la bras Cla ve: Mo de lo de co mu ni cación Lass well, Ba ses de da tos de imá ge nes.

\section{Jorge CALDE RA-SERRANo \\ FE LIPEZAPICO-ALONSO \\ LASSWELL COMMUNICATION FORMULA AS A METHOD TO IMMPLEMENT \\ DOCUMENTARY DATA BASES IN AUDOVISUAL MEDIA}

\begin{abstract}
Laswell's com mu nica tion model is the one that has been ac cepted by the journalists'communityasagood formula of com municative relationship
\end{abstract}

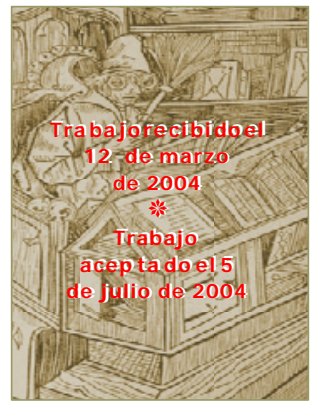


with the TV spec ta tor. The model sets forth the need for the jour nal is tic message to solve a se ries of ques tions im plied in the five "Ws": what, how, when, where and why, which include the communicational elements that must be made evi dent in a pro cess which emits and re ceives. The same method is also ac cepted in the audio vis ual com mu nica tion me dia and for that rea son seems ade quate to im plement docu men tary data bases. There fore, if the audio vis ual information piece is constructed by answering to the questions made by Laswell's method, it would be ap pro priate to dis mem ber again such in forma tion and pre serveit and store it in the data bases by means of spe cific fields.

Keywords: Lass well's Communication model, Ima ge da ta bases.

\section{INTRODUCCIÓN}

A unque vilipen dia da, la te le vi sión ha sidounins tru men tovital para el de sa rrollo 1 comunicativo de la sociedad actual en las últimas décadas, un elemento vertebrador e integrador del pensamiento colectivo inicialmente nacional y que posteriormen tehaevolu ciona dogra cias a las tec nologías delas tele co munica ciones hacia la idea de "alda gdbal" profetizada por MacLuhan ${ }^{1}$ en su obra clásica.

La te le vi sión cuen ta con la ne ce si dad deve ri ficar, con tex tua li zary apo yarla producción que conforma la parrilla programática de la cadena, para lo cual utiliza los servicios de documen ta ción con quela to talidad delas ca denas na cionales yregionalescuen tan en tresus orga nigramas. Es ne cesario con tarconlages tión co rrec tayválida de información so no ra, vi sual, tex tual y au dio vi sual para po der apor tary op timizarla documen ta ción necesa ria solicita da porlos usuarios, yalcanzarasílos objetivos delacadena, tantodeexplotacióncomoeconómicos.

Enelmarco deestas empresas au diovisuales es necesarioimple mentary desarrollar las herramientas documentales válidas para conseguir los objetivos señalados conanterioridad, por lo que pa rece ade cua do el in ten to de plas mar en di chas herra mientaslas fórmulas deconsultaylas necesidades delosperiodistas.

Aten dien doa di cho fac toryalanece si dad de que elusuariocuenteconlain formación solicitada en el mar co de una rea li dad em pre sa rial, se cree opor tu no que la implementación de las bases de datos documentales sean un fiel reflejo de la fórmula que emplea el perio dis ta para ofrecerlein forma ciónal teles pec tador, en el mar co de un pro cesoco mu nica tivo. Es poresarazón, porlo que se de sea mos trar los dis tin tos modeloscomunicativos ydarlevalidezalaceptadoporelpresenteartículo,lafórmula de Lass welly, más con cre ta men te, "las re glas de las cin co W"2 emplea das a partir

1 Marshall Mcluhan, La gglaxia Gutenbeg génesis dd “homo typographicus”. Barcelona: Círculo de Lectores, 1993. 1962 fue el año de la primera edición de dicho libro.

2 Se re fie re a las cin co Ws de las que se ha bla en in glés: who, what, when, why and whe re (quién, qué, cuando, por qué y dónde) N. T. 
de Lasswell como método válido para desarrollar las bases de datos documentales, especialmenteelmaterialaudiovisual, queesen elquesecentraelpresentetrabajo.

\section{PRINCIPALES MODELOSDECOMUNICACIÓN}

Eles tu dio de laco mu nica ción es bas tan te complejo so bre todo porla plu ralidad del pro pio fe nó meno, lo que hacene ce sa ria la crea ción de re glas para en ten derla co municacióny poderanalizarsuscausasyefectos. Lacomunicación puedeestablecerse en tre dos perso najes aun que no exis ta un có digo ad mi tido y acep ta do porlos dos sujetos; idéntica comunicación se realiza entre la televisión y los telespectadores, aunqueconunaseriedecaracterís ticaspropias.

La comunica ciónen tre per sonas haido evolucio nan do alolargodel tiempoconforme ha evolu ciona dola cienciayla técnica dela transmisión delain formación, yh a superadobarrerashastahacebien pocoinsalvablescomolasdetiempoyespacio.

Todoslosmodelosdecomunicaciónuniversalmenteaceptadoscuentanconcua troelementoscomunes:elemisor, el receptor, elmensajeyelcanal.

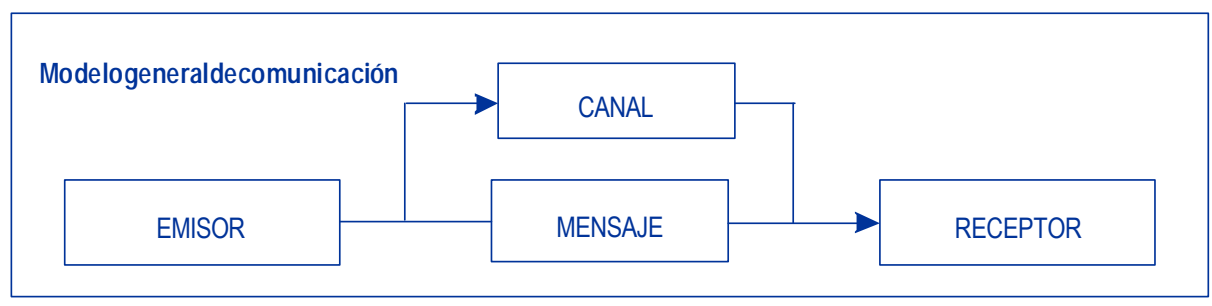

Serealiza elproceso decomunica ción en elmomen toen quese trans miteunmensa je que va delEmisara un Reeqtor por me dio de un canal que pue da ser utiliza do por ambas partes. Igualmente es básico para que se realice una comunicación correcta que tan to elEmisarcomo el Reeqtorcom par tan un mis mo ćdigo Por lo que al es quemaanteriorsehabrá de am pliar ein cluirun códigoco mún en treEmisor y Reeqtar.

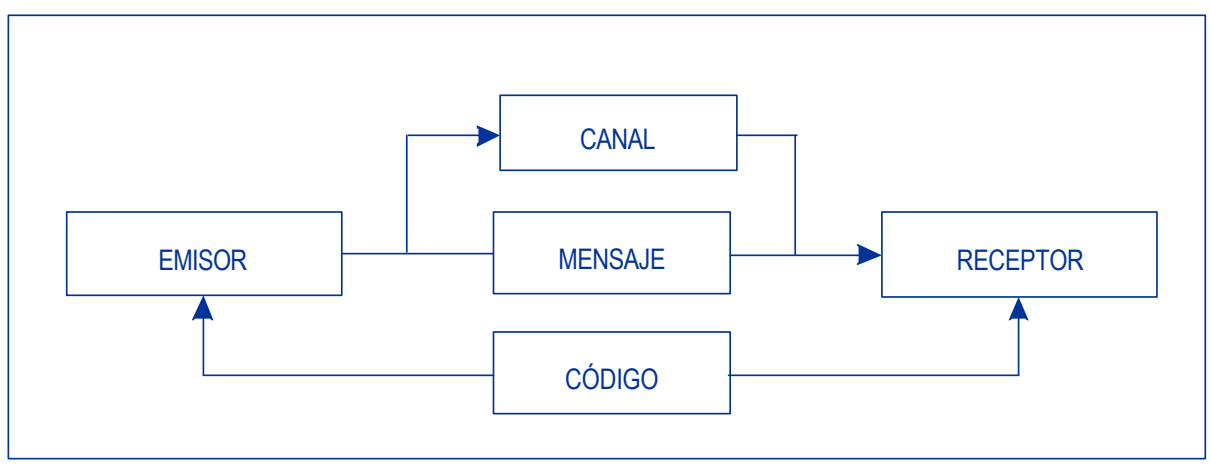


$\mathrm{Al}$ igual que el ćdigg el camal es un fac tor vital para la bue na mar cha del pro ce so comunicativo. Joseba Zúñiga ${ }^{3}$ divide la comunicación en directa y mediata. Directa es aquella que no necesita ningún medio tecnológico entre emisor y receptor; es decir, existeunacomunicación directaconrela ciónendirecto. Porelcontra rio, lacomunicación me dia da es aquel la en la cual son ne ce sa rios ca na les téc ni cos para que ésta exis ta. Lacomunicación mediadaeslautilizada porlos diferentes mediosdecomunicación y laque,comoseindicabaconanterioridad, superalapruebadeltiempoydelespacio.

\section{Modelo Aristotélico}

Unodelosmodelosqueanalizanla comunicación es elmodeloaris to télico, elcual basa su es tu dio en la co mu ni ca ción oral. Es evi den te que este mo delo tan sólo cu bre parte de la casuística de los modelos de información y obviamentenocontemplael proceso comunicativo tal y como se organiza en los medios televisivos, aunque sí cuenteconunapartediscursivaquepodríaserexportablea dichomodelo.

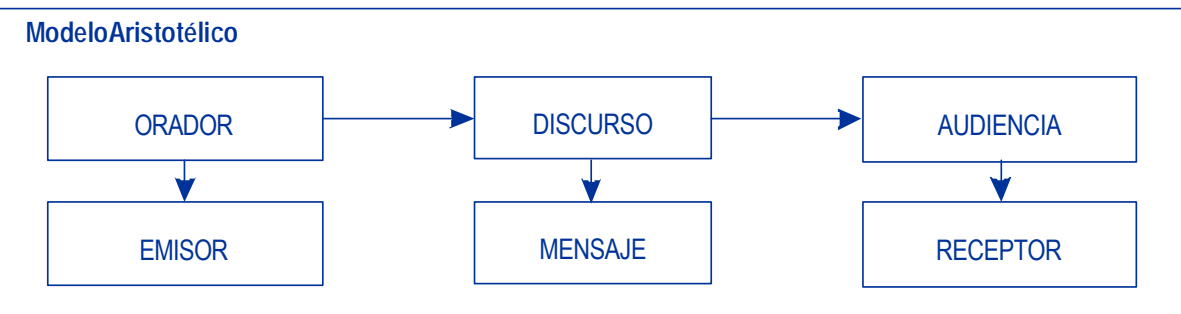

\section{T eoría matemática de Shannon ${ }^{4}$ y Weaver}

La teoría matemática de la comunicación fue formulada por Shannon en 1949 y desarrollada con posterioridad por Weaver analizan do el cam podelas tele co mu nicaciones. Shannon basa su información en que un estímulo exterior debe transformarse en una en tra da de in for ma ción en el sis te ma de co mu nica ción, y tam bién incluye la reacción del receptor por medio de una respuesta al mensaje. Teresa Aguirreazaldegui ${ }^{5}$ habla de "[...] una teo ría del ren dimien to in formacio nal o trans misiónóp timadelosmen sajes". Elobjetode esta teo ría dela comunica ciónesaporta da por Aguirreazal degui to man do unacita de Wolf" "[...]mejorarla velo cidad delatransmi sión delos men sajes, dis mi nuir las dis tor sio nes ylas pér di das de in formación, aur mentarelrendimientogeneraldelprocesodetransmisióndeinformación.”6

3 Jo se ba Zú ñi ga. CommicadónAudiovisual. San Sebastián: Es ci vi, 1998. pp. 79 y ss.

4 Clau de E. Shan non. Teeńa matemática dela commicacón Ma drid: For ja,1981.

5 Teresa Agirreazaldegi. Elusodeladbammtacónaudiovisual enlosprogramasinformativosdianiosdetelevisón Bilbao:Universidad del País Vasco, 1996, p. 151.

6 Mau ro Wolf. La inmeticaión dela commicaión demasas Barcelo na: Pai dós, 1991. 
El desarrollo del modelo matemático comunicativo de Shannon comienza en el mo men to en el que la fuen te de co mu ni ca ción ge ne ra un men saje, esta fuen te se lec ciona el men sa jey el trans mi sorloco difica, con vir tién do la enunase ñal capaz de ser trans miti da por un ca nal in formá tico, el cual será el vehícu lo para en viarlain forma ción des de untrans misordein forma cióna otro apa rato re ceptor; estecanalpue detenerruido porin terferen cias de comunicaciones ex ternaso por ele men tos defectuososdelsistema.

TalycomoseñalaAntonioHernández:

[...]Mien tras que Lass well se ocupa del con tenido delos men sajes, la teoría de Shannon prescinde prácticamente de él para ocuparse, desde una perspectiva más tecnicis ta, y has ta cier to pun to, más eco no micis ta, sólo delcontinentedelosmensajes, evitando in te resarse porel con tenidode los mis mos. Lo que in te re sa a este mo de lo no es co no cer, ni mu cho menos des cribiro in ter pre tar los men sa jes, nien su fase de emi sión ni en su fase de recep ción, sino es tu diarlos proce dimien tos quelogren au men tar la eficacia del proceso informativo. Shannon intenta lograr, mediante el estudiodeíndices de ruido, silen cio, en tro píao re dundancia, en treotros, la mayoreficaciaposible, en unsentido técnicoyeconómicodelproceso de transmisión (emisor - recepción) de la información. ${ }^{7}$

Elhe cho deque el modelodeShannon seolvidedel contenidodel men sajeyvuelque sus es fuerzos en elcon tinen te ha sido uno delas acu sa ciones más repe ti das hacia dichomodelo.

Estees quemade co mu nica ción, surgidoa partirdeles tu diodelas tele co mu nicaciones, ha sido adaptado para el campo de las comunicaciones humanas y resulta muyútil paraanalizarlosmedios decomunica ciónal to maren cuenta elanálisis delos efec tos que producen losmassmædaa través delacomunicación. ${ }^{8}$

Umberto Eco, en el año 1972, explica así el modelo de Shannon para analizar el procesocomunicativo:

[...] existe siempre una fuente o manantial de la información, desde la cual a tra vés de un apa ra to trans misor, es emi ti da una señal; esta señal via ja a tra vés de un ca nal a lo lar go del cual pue de ser in ter fe ri da por un ruido. Al sa lirdel ca nal, la se ñal es re co gi da por un re cep tor que la con vier te enunmensaje.Comotalel mensaje escomprendidoporeldestinatario. ${ }^{9}$

Teo ríamate máticadeShanonyWeaver

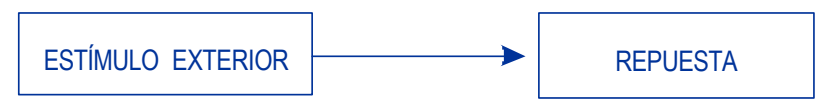

7 An to nio Her nán dez Pé rez. Doamentacónaudioisual:m todbloǵa para d análisis doamental delainfor macón periodística audiaisual. Madrid: Universidad Com plu ten se, 1992, pp. 29 - 30.

8 Para am pliarin for ma ción so brela teo ría ma te má tica de Sha non ver:JoséMa ría De san tes Guanter. Teena y régimenjuńdicodladbamentadión Ma drid: Eu de ma, 1987, pp. 229 y ss.

9 Um ber to Eco (dir). Estetica eteeniaddl' informaziones Mi lan: Bom pia ni, 1972, p. 127. 


\section{T eoría y modelo de Maletzke}

El psicólogo alemán Maletzke, especializado en psicología de la comunicación, formulaen1963elmodelocomunicativoqueesconocidoporelnombredelautor.

Modelode Maletzke

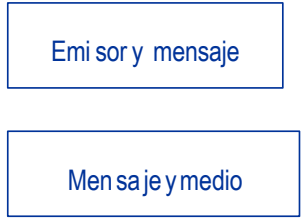

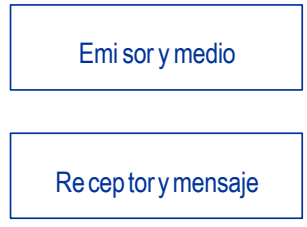

Emi sor y receptor

Recep torymedio

Estemodelodecomunicaciónes tablece seis diagra masy desa rrollalassiguientes relaciones:

* Relaciónentredenisarydmensaje Ana li za el pro ce so de crea ción del men sa je, y estudia la intención de éste.

* Relaciónentredemisarydmedio Es tu diala in fluencia dela em pre sa co mu nicati va sobre el medio, sobre todo desde el punto de vista tecnológico.

* Relaciónentredemisarye receptor. Está influenciada por la autoridad del emisor, la imagen que el re cep tor guar da del me dio, los po si bles con tac tos en tre emisor y receptor, la predisposición frente al medio, las relaciones emocionales entre emisor y receptor y, por supuesto, el prestigio del emisor.

* Relacónentredmensajeydmedio De ter mi na que la no ti cia sea ofre ci da de manera diferen te se gún se tra te de pren sa, ra dio o tele visión. El me dio au dio visual cuenta con la ventaja de que la imagen puede trabajar de manera mu chomás directa las emociones.

* Rdacónentred reeqtoryd mensaje Consiste en el hecho de que la información cumpla o no las aspiraciones y necesidades del receptor.

- Relacónentrødreceptorydmedio Surge de la relación entre el medio y el receptor, sobre todo tomando en cuenta el prestigio de dicho medio.

\section{Teoría H ipodérmica}

Esta teo ría sur ge con la ex plo sión de los me dios de co mu nica ción de ma sas y tras ana lizarlos efec tos produ cidos porlos me dios en la so cie dad. Fue ela bora da a par tir de la psi cología con duc tis ta, la cual parte de laidea del es tímulo como fór mu la para llegaraunarespuesta.

Esta teo ría analizalas reac ciones de cadauno delosin dividuos, to ma dos porseparado, una vez que los medios de comunicación ofrecen una información, la mayor parte delas ve ces in fluen cia da porés tosmismos. Los au to resqueana lizan esta teo ría 
hipo dérmica son WrightMills ${ }^{10} \mathrm{y}$ Wolf. ${ }^{11}$ Debeque dareviden cia do que di cha teo ría se encuentra poco desarrolladaademás de con tar con un alto gra do de re cha zo por partedeloscomunicólogos.

\section{Modelo Comunicativo Semiótico}

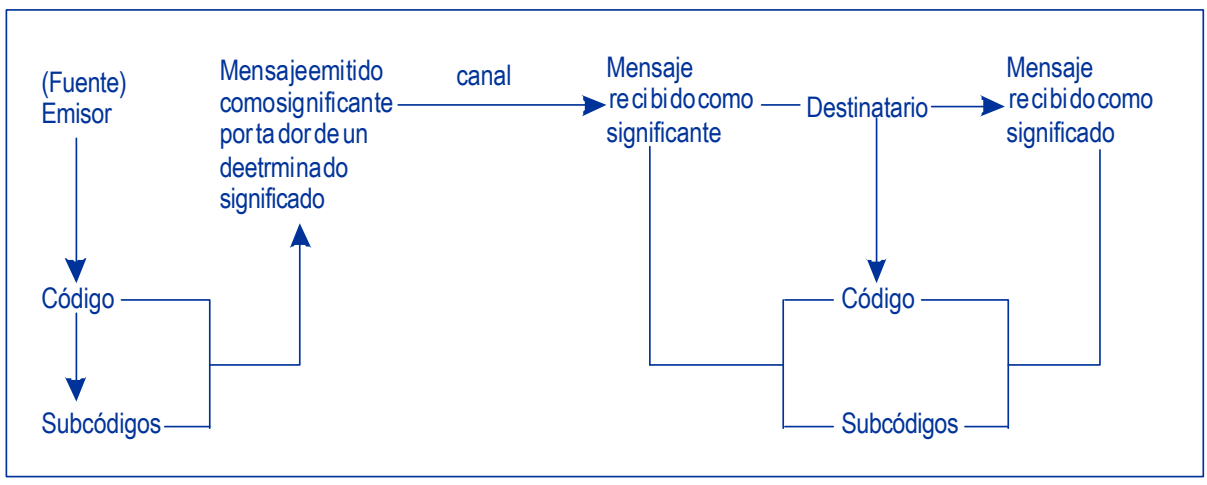

Esteesquemanos muestraelmodelocomunicativosemióticomostradoporUmberto Eco, ${ }^{12}$ quien señala lain fluen cia de di fe ren tes dis ciplinas en el para dig ma dela comunicación e introduce factores eminentemente semánticos, tal y como señala Mau ro Wolfal men cio narqueel có digo se en tien de comola co rrela ción en trelos elemen tos de sis temas di ferentes; es de cir, elcó digo “[...]garan tizalapo sibilidad de dichatransformación".13

Wolfnosmues tralaimportan ciaque tienela des co dificación delain formaciónque haceuncolec tivo alcual va dirigidalain for ma ción dema sas. Igual mente señala que es diferenteelsignifica do delmen sajeemitidoyelsignificadodelmensajerecibi do cuandohayunaincorrectadescodificacióndelmensaje.

Por lo tanto la comunicación contiene dos ele men tos bá si cos que han de en tenderseno sóloutilizan do un mis mo código, sino des codifican do deidén ticamanera los có digos envia dos por unos y recibi dos porotros. Lain ten ción del emi sorala hora de emitir el mensaje ha de ser descodificada con la misma intención por parte del usuario.

10 C. Wright Mills. Power pditicandpeeple New York: Oxford University Press, 1963.

11 Mau ro Wolf. Lainuestigacóndela commicaaóndemasas Barcelona: Paidós, 1991.

12 Hum ber to Eco [et. al.]. Primapropostaperunmodilodiricercainterdisaiplinaresul rapportotelevisione/ pubblicoPerugia:Ins ti tu to diEtnologíae An tro pología Cul tu rale, 1965.

13 Mau ro Wolf. Op at p. 135. 


\section{Modelo semiótico-textual}

Este mo delo se ña la que para una bue na comu nica ción nose debe teneren cuenta únicamente la correcta transferencia del mensaje sino que habrá que considerar las relacionesprácticastextuales.

Taly como se ña lan Eco y Fabbri, ${ }^{14}$ hablarde una co rrec ta co municación simpliffcán dolo todo a una co rrec ta des co di fica ción pue de serun erroral no tenerencuen ta unaserie de cues tiones, tales comoque el receptorno recibemen sajes sinoconjuntos textuales, que estos mensajes no son comparados con otros, y que los mensajes enviadosporelemisornosonúnicos.

El emisory el re cep torno coin ci den con la fuen te y el des ti na ta rio de la teo ría semiótica, ya quehabitualmentetantoemisorcomoreceptorsonentendidoscomodos partes del modelo comunicativo sin ningún tipo de intención, mientras que para la teoría semiótica ambos polos sugieren y posibilitan la dinamización del proceso al haberinteractuaciónentreemisoryreceptor.

\section{TEORÍA Y MO DE LO DE COMU NICACIÓN DE LASSWELL}

Ha roldLass well(1882-1978) des ta ca porserdelos prime rosin ves tiga do res que ana li zan elefec to quepro vo canlos me dios enlos elec to res. Lass well plas malas bases dela teo ría fun cio na lis ta, que se ad hie re a la teo ría de los efectos, aun que apor ta ciertas par ticularida des del proce socomunica tivo ydelos efectos so brelos mensajes, y señalaquelosmensajessiempreproducenunefectoenquienlos recibe.

El modelo comunicativo de Lasswell también es conocido como paradigma de Lass well, elcual fue di fun di doen 1948 en forma dear tículo "Es truc tu ray función de lacomunicación de masas". Lasswellapoyasumodelocomunicativoanalizandoestudios políticos, de opinión pública y sociales. En este artículo trata de explicar el comportamientodelasmasasen respuestaaunosestímulosdeterminadosofrecidos por el emi sor. Esta idea da pie para su más co no ci da teo ría, que in clu ye las pregun tas que han de llevarse a cabo paraana lizarein terpre tarun acto de co munica ción, tales como ¿quién lo dice? ¿qué cosa dice? ¿en qué y por qué medio lo dice? ¿a quién se lo dirige? ¿cuál essu finalidad? Estemodeloviene en partederiva do del mo deloaris totélicoenunciadoconanterioridad.

ParaLasswellcualquierprocesocomunicativocuentaconlossiguienteselementos:

* Quién: que se co rres pon de con el emi sor, por lo cual se es tu dia la pro ce dencia del mensaje.

* Qué: que correspondería con el mensaje, y servicio para hacer el análisis de contenido.

14 Humberto Eco; P. Fabri, Progetto di ricerca sull'utilizzaziones dell'informaziones ambientale. Problemi dell'informaziones, 1978, no 4, pp. 555 - 597. 
Porqué me dio o quéca nal:correspondería al ca nal en el mo delo clá sico, lo cual ayuda a analizar los medios de comunicación.

* A quién va dinigida la comunicación: sería el receptor, lo que permitiría realizar diferentes estudios de la audiencia.

* Con qué efectos: analiza no tan sólo el mensaje sino lo que éste produce.

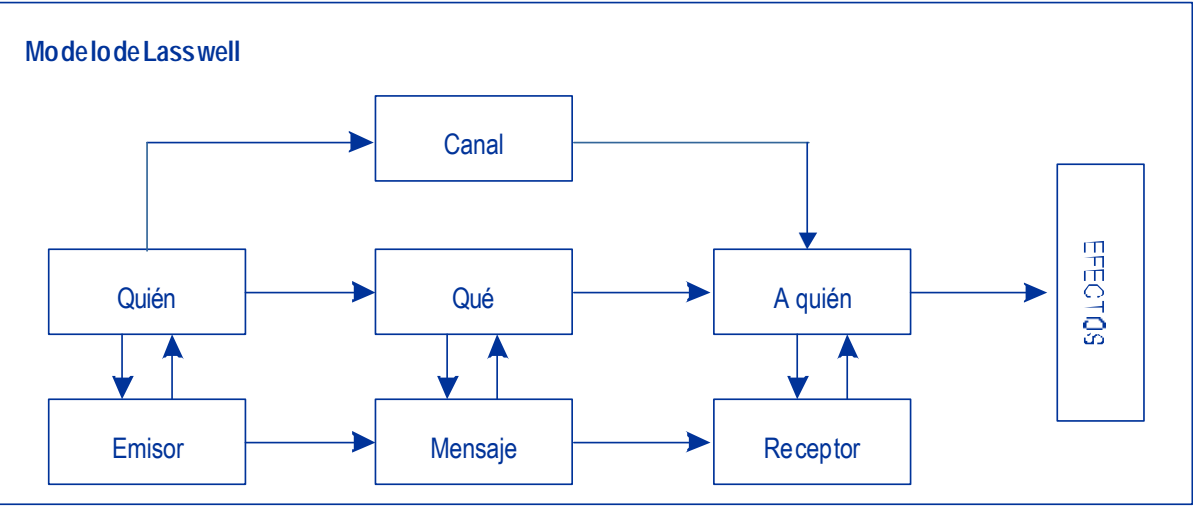

Estemodelocomunicativohasidoadaptadoalos mediosdecomunicaciónyservidodeguía para mu chostrabajos.Zúñigaseñalaun po sible as pec toquenocontempla este mo delo co mu nica tivo, lo cual no es váli do para de sa cre di tar dicha teo ría.El pa radig made Lass well es uni direccio nal, no hay una posiblein terac ción conel teles pectador, tan sólo ana liza los efec tos quelos men sa jes produ cen so bre éste, tan toindividual como colectivamente, pero no ofrece la posibilidad de dar respuesta a los efectosplanteados.

Dicha teoría y fórmula co mu nica tiva ha sidoacep tadaporlos me dios de co mu ni cación para confeccionar la información. Esta teoría monta la pieza informativa aten diendoalas preguntasplantea das porLass wellensufórmula comunicativa,con el fin de pro du cirun efec to de riva do dela po lítica em pre sa rial del me dio y de la ideología deéste. Dicha formula ha sido plan tea da muy es pecial men tepara el ma te rialde informativos, pero es igualmente útil tanto para documentaciónen prensaes critay radiofónicacomoparalaaudiovisual.

\section{LAFÓR MU LADE LASSWELL Y LAS BASESDE DATOSEN MEDIOS AUDIOVISU ALES}

Yasehacomentadoqueprácticamen tela to talidad delos manuales periodísticos to man comoválidala fórmula deco munica ción de Lass wellparain terrela cio narsey comunicarseconsuaudiencia.Porlotanto, lasin formaciones periodísticasintentan darrespuestaacadaunadelaspreguntasplanteadaspordichafórmula. 
La idea del desarrollo y optimización de las bases de datos documentales de los medios au dio visuales es ex tre ma da mente sen cilla, lo quenoleres ta valory síle ofrece legitimidad a sus resultados desde el momento en que tanto el principio como el fines el propiousua rioy la rela ción de éste conlas herramien tas docu men tales. Elperiodista-usuario no es un ser inactivo, un ser inerte que obtiene información de los departamentosdedocumentaciónde formapa siva, sinounente vivo quein te rac túa de manera muy diferenteconeldepartamentodedocumentacióndependiendodel in dividuo. Estose tra du ceenla ne cesidad de crear he rramien tas marcosygenerales para que cada periodista pueda acceder a la información de la manera más cómoda sinolvidarloscriteriosdepertinencia, relevanciayexhaustividad.

Porlotanto, sielperio dis taorganizalain formación televisivaqueposteriormente se di fun de, to man do en cuen ta las cues tiones plan tea das por Lass well, debe con seguirunaherramien tado cumen tal capazdedescomponerdichos elementos encampos de la base de da tos para po der asíre cu pe rar el to tal de lain for ma ción, o par te de ella, con base en ele men tos con los que fue ron crea dos. Para ello es ne ce sa rio do tara eseperio dis ta de una es truc tu ra de base de da tos abierta y fle xible, ya que el tipo de solicitudes del usuario puede variar atendiendo a las modasinformativas, ${ }^{15}$ aunque este cambio sí determina más la ges tión y el análi sis docu men tal quela propia es truc tu ra delabasededatos.

Unaherramientadocumen talau diovisualdebecon tarconotrain formaciónqueno sealapuramenteinformativa, aspectos relacionadosconlos derechos de explotación y reutilización, características físicas del soporte y de la emisión en sí misma, etcétera, todolocual se es capa del pro ce so co mu nica tivo marca do por Lass well y hace necesa riosucontrolenelmarcodelasinstitucionesdocumentalesaudiovisualestelevisivas.

Se ofre cen a con ti nua ción las áreas con las que debe con tar una base de da tos para lagestión dein formación au diovisual en los servicios de in forma ción ydocumentacióndelascadenasgeneralistas. ${ }^{16}$

15 Sedenominamodain formativa ala evolu ción en el tipoy la fór mula de ejecu ción del assolicitudes de los usua rios. La ac tua li dad y la rea li dad cam bian te, tan to de los mé to dos como de los hábi tos en nuestra sociedad, hace necesario estar alerta a los nuevos requerimientos de los periodistas que vendrán determinados por las solicitudes de los telespectadores, por lo que aquello que era una mera anéc do ta o cu rio si dad po dría con ver tir se en he cho no ti cio so o in te re san te.

16 Da tos ex traídos de la te sis doc to ral de Jorge Cal de ra Se rra no que bajo el título "Ardhivosdetelevisión: nuevaspespeetivasmetodbóǵcas para \& tratamientodbaumental delaimagenenmovimientoenprogramasinfor mativos' fue de fen di da en la Uni ver si dad de Sa la man ca, en la que se plas ma un no ve do so mé todo de gestión documental para los archivosdetelevisión. 


\begin{tabular}{|l|l|}
\hline \multicolumn{2}{|c|}{ Base de Datos } \\
\hline \multirow{4}{*}{} & Control \\
\cline { 2 - 3 } & Descripción física \\
\cline { 2 - 3 } & Designación \\
\cline { 2 - 3 } & Descripcióndocumental \\
\cline { 2 - 3 } & Descriptores temáticos \\
\cline { 2 - 2 } & Descriptores onomásticos \\
\cline { 2 - 3 } & Descriptores geográficos \\
\cline { 2 - 3 } & Fecha \\
\cline { 2 - 3 } & Producción \\
\hline & Emisión \\
\hline & Localización \\
\cline { 2 - 3 } & Responsabilidad \\
\hline & Miscelánea \\
\hline
\end{tabular}

De for ma ge ne ral, cabe se ña lar que exis ten áreas que no son váli das para res ponder a las cuestiones plan tea das por Lass well pero que la in for ma ción apor ta da en el área de control es necesaria para determinar la producción y la cali dad de ésta. Otro ejemplo de ello son los datos relativos a la descripción física, que en nada ayudan a describirelcontenidopero síelcontinentedocumental;aspectos relacionadoscon la producción, la emisión, y la localización del material en los depósitos. Ni siquiera la responsabilidado cua dro técnicodelmate rialaportain forma cióndes de el puntode vis ta delos da tos que componen el acto co mu nica tivo, aun que sean ne ce sa rios para algunoselementos delcontenido,yválidos paralare cuperación porsímismosoutilizadosjun to a otroselementosporme dio delosoperado resbodeanos

Vanades cribirseaquellasáreasqueofre cencontes ta ciónal Para digmadeLasswelly sehará finalmenteun esquemadeloscamposypreguntasresueltasportalesáreas.

\begin{tabular}{|l|l|}
\hline \multicolumn{2}{|c|}{ Área de Designación } \\
\hline \multirow{4}{*}{} & Título documental \\
\cline { 2 - 2 } & Subtítulo documental \\
\cline { 2 - 2 } & Título programa \\
\cline { 2 - 2 } & Título serie \\
\cline { 2 - 2 } & Título de las partes \\
\cline { 2 - 2 } & Título original \\
\cline { 2 - 2 } & Subtítulo \\
\cline { 2 - 2 } & Núme ro en la se rie \\
\hline
\end{tabular}


En elárea de de signa ción se ofrecein for ma ción sobre el tí tu lo o títulos ofre cidos porla empresa, asícomoel títulodocumentalaportadoporelpersonaldelserviciode documentación, en caso de que di cho ma terial no ten ga un título atribui do. Esto es una reali dad cuandose analizan piezas deunin formativo, aun quepara progra mas de en tretenimien to oproductos completosytermina dos es válidoeltítuloofrecidop or lacadena.

\begin{tabular}{|l|l|}
\hline \multicolumn{2}{|c|}{ Área de descripción documental } \\
\hline \multirow{2}{*}{} & Resumen \\
\cline { 2 - 3 } & Análisis cronológico \\
\cline { 2 - 2 } & Análisis cronológicooriginales \\
\cline { 2 - 2 } & Condicionesatmosféricas \\
\cline { 2 - 2 } & Obras artísticas \\
\cline { 2 - 2 } & Descripciones externas \\
\hline
\end{tabular}

El área de descripcióndocumental es especialmenterelevante en el marco de las empresas audiovisuales desde el momento en que hay campos sólo existentes en el análisis y tratamiento de la información audiovisual. Puede observarse en el cuadro cómo se cuen ta con el cam po de aná li sis cro no ló gi co en el cual se des cri ben las imá genes, secuencia porsecuen cia, men cionandoen primerlugarelcódigodetiempode inicio de las imágenes, el plano y el movimiento de la cámara, y terminando con la descripcióndelaimagenentextolibre.

Ejemplode ellopodríaser:

00:00:32PGz.inPMi7 DedaracionesennuedadgprensadeJoséManáaAznar,presidantedl gobiem, señalanddlaneesidaddereamuerospuestosddrabaja

Ade más de di cho cam po, se ob servan otrosigual men te váli dos, como elde obras artís ticas, en elqueseseñalanlas diferentes piezasmusicales, es cultóricas, pictóricas, et cé tera, que sevenya las cuales hace re fe ren cia eldo cu men to, ylaiden ti fica ción de lasdiferentescondicionesatmosféricasquepuedanobservarse.

\begin{tabular}{|l|l|}
\hline \multicolumn{2}{|c|}{ Área de descriptores temáticos } \\
\hline \multirow{3}{*}{} & Tema \\
\cline { 2 - 2 } & Plano tema \\
\cline { 2 - 2 } & Denotación temática \\
\cline { 2 - 2 } & Identificador \\
\hline
\end{tabular}

17 Pla no Ge ne ral con un mo vi mien to de cá ma ra, en este caso, un zomin para acer car se a un Pla no Medio. Se describe por lo tan to un mo vi mien to de cá ma ra y con pos te rio ri dad selle va a cabo la des crip ción delasimá ge nes. 
Otraáreaimportanteenlades cripción deinformación audiovisualeslaidentifica ciónde as pec tos te máticos tan to de ele men tos que seven comoalos que se hace re feren cia. Esta dua li dad del aná li sis en la que se dis tin gue en tre lo que se ve y a lo que se hace re fe ren cia es un ele men to di fe ren cia dor de este tipo de ges tión do cu men tal, lo que que da rá plas ma do en otros cam pos. Igual men te se ofre cein for ma ción so bre as pectos denotadostemáticamenteya que suele ser habitualrealizar las consultas por me dio del tipo de atribu tos que ofre celain for ma ción; es de cir, de unalec tu ra subje tivaso breelacon te cimien to, he chooimágenes y no sobreloque sevecla ra men te enla piezaaudiovisual.

\begin{tabular}{|l|l|}
\hline \multicolumn{2}{|c|}{ Área de descriptores onomásticos } \\
\hline \multirow{2}{*}{} & Persona \\
\cline { 2 - 2 } & Planopersona \\
\cline { 2 - 2 } & Denotación de personas \\
\cline { 2 - 2 } & Entidad \\
\cline { 2 - 2 } & Planoentidad \\
\cline { 2 - 2 } & Denotación de entidad \\
\cline { 2 - 2 } & Autor \\
\cline { 2 - 2 } & Intérprete \\
\cline { 2 - 2 } & Colaboradores \\
\hline
\end{tabular}

Otra área interesantey primordial es la relacionada con los elementos onomásticos en la que se identifican tanto los nombres de personas físicas como jurídicas; como pue de ob servar se exis te una cla ra di feren cia en los cam pos en trelas per sonas físicas y las perso nas ju rí di cas, ha cien dola mis ma dis tin ción que en elárea te má tica entreinformaciónvista, referenciadaydenotada.

Aes tos ele men tos porme diodeloscualeses habitualrecu pe rarlain forma ción en las televisio nes, se une otra in forma ción váli da para la descrip ción deobras artís ticas, como sonlosau tores ein terpretes (se re cuer da que el tér mi no obras ar tís ti cas está toma do des de un pun to de vis ta muy general). Enúl timolu gar se ofre cela po sibilidad de in cluir a los co la bo ra do res que han par ti cipa do en la pues ta en es ce na de los programastantodeinformativoscomodeentretenimiento. 


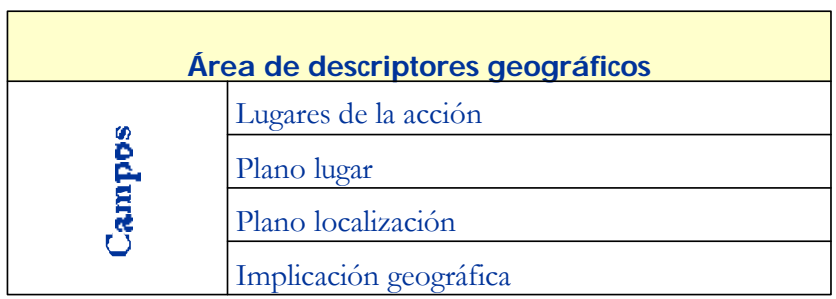

Junto alaidentifica ción de personasy temas, el círculose cierra alseñalarloslugares vis tos enlain forma ción, ya seangenerales (planolugar) yaseanespecíficos (plano localización), y lasciu da desozonas geográficasenlas que se desarrollanlosacontecimien tos. Enúl timolu gar tam bién seiden ti fican los paí ses o con jun to de paí ses con nombreformalsobrelosquerepercutelainformacióndeunamaneradirecta.

\begin{tabular}{|l|l|}
\hline \multicolumn{2}{|c|}{ Área de fecha } \\
\hline \multirow{3}{*}{} & Noticia \\
\cline { 2 - 2 } & Periodo noticia \\
\cline { 2 - 2 } & Grabación \\
\cline { 2 - 2 } & Acontecimientocíclico \\
\hline
\end{tabular}

Enlapresen teárea se des cri ben ele men tos cro noló gi cos, como cuán do se ha desarrollado la no ti cia, ya sea un día con cre to o com pren dido en tre dos fe chas, ola fechadegrabacióndelasimágenesvistasenlapiezainformativa.

\begin{tabular}{|l|l|}
\hline \multicolumn{2}{|c|}{ Área de responsabilidad } \\
\hline \multirow{3}{*}{} & Director / Editor \\
\cline { 2 - 2 } & Realización \\
\cline { 2 - 2 } & Cuadro técnico \\
\cline { 2 - 2 } & Plano reporteros \\
\hline
\end{tabular}

Aunquepuedenoserespecialmenterelevantepararespondercuestiones delpara digmaestaáreaidentificaquiénharealizadoeldocumentoaudiovisual.Debequedar re fleja do aquíque los res pon sables de una pieza de unin formativono sonúnicamentelos re por te ros en carga dos de la con fección de la ban daima gen o del so nido, la autto ría de este ma terial es compartida por todo el perso nal, in clui dos los pro fe sionales delserviciodedocumentaciónque ofrecentan toin formación tex tualcomoau diovi sual para la confección de la pieza(información textual para la contextualización, y audiovisual paraofrecertantorecursosparcialescomototales). 


\section{CORRE SPON DEN CIA DE CAMPOSY CUESTIONES PLANTEADAS POR LASSWELL}

Unavezdescritademanerasucintatan tola fórmuladecomunicacióndeLass well comounabase de da tos es tan da riza da, selleva ráa cabo la co rres pon den cia en tre dichos campos y las preguntas de Lass well, lo que re ve la rá que para que di chas he rra mien tas sean valida das yve ri fica das de ben ha bersido im ple men ta das para res ponderadichoprocedimientocomunicativo.

Elma terial televisivo taly como es pre sentado enlosin forma tivos tipo no ticiario cuen ta dehe chocondos mo dalida des diferen tes parain terpretar dichopro ce so comunica tivo.Laprimera de ellas está en cua drada den trodela es truc tu ra ge ne ral dela programación de la cadena, y más concretamente del propio noticiario, por lo que parte de la cadena comunicativa será siempre la misma, como el emisor y receptor $($ emisor $=$ medio televisivo; receptor $=$ telespectador $) ;$ sin embargo la unidad de análisis y tra ta mien to, asícomolo de emisión, es la noticia delin forma tivo, porloque será en elmarco dedicha es tructura con cep tualen el que se analiza ráy proce sarálainformaciónaudiovisualconbaseenlafórmulaplanteadaporLasswell.

Porlo tan to para simpli ficarel mo delopa ra digmático delensa yis tava mos airseñalandocadaunadelascuestionesplanteadas ycuáles sonloscampos primitivamenteseñaladosqueofrecenrespuestasaellas.

\section{Quién (quién, a quién = emisor)}

Determinarelquiénesmu cho más amplio que fijar elemi sordelproce so co mu nicativo,sindudapuedenderivarsediversosfactores. Estono sólo suponemarcaralemisorde la información, en definitiva el sujeto agente, sino que asimismo habrá que determinar so bre quién re cae lain forma ción, a quién bene ficia o a quién perju dica; en de finitiva, tal comoseñala GarcíaGutiérrez, habráquedeterminarelsujetopaciente. ${ }^{18}$

El quién pue de aún serampliadocomounderivado dela naturaleza audiovisualdel men saje tele visivo, y dis tinguiren tre quién rea lizay re cibela ac ciónen trelas personas físicasyjurídicas vis tas, yentrelasentidadesypersonasalasquesehacereferencia.

Porlotanto,yaten dien doala dobleinten ción comunica tivaseñala da con an terioridad, el quién que da rá refle ja do porlos cam pos des tina dos al cua dro técnico; es de cir, di rec tor, realiza dory cua dro téc nicoengene ral, y el marco del pro piomen sajeinformativo. Excluyendo el proceso intencional del medio, se contaría con los destinadosenloscamposdepersonasfísicasyentidadesvistasyreferenciadas; es decir,las perso nas, laen tidad, el pla noperso na, el planoen tidad, el au tor, elin térpretey loscolaboradores.

18 An to nio García Gu tiérrez.ProcedimientosdkenálisisdbamentalautomáticaEstudiodbcasa Se villa: Ins ti tuto Andaluz del Pa tri mo nio His tó ri co, 1996.p. 69. 


\section{Q ué (qué se dice = mensaje; efectos)}

Más complicado de describir en cualquier base de datos es el proceso de desencripta ción del contenido delmen saje. Dichades crip ción te má ti ca sellevaa cabo tradicional menteporme diodelain dizaciónylaclasificación to man doencuentael tipo deunidad docu mentalyla organiza ción delain for ma ción. Identi ficar elquére flejadoporLasswellayudaaidentificarlos contenidos te máticos delmensaje.Para García Gutiérrez ${ }^{19}$ estemensa je vie ne com pues to por unase riede ele men tosquelocom ponen como son la acción, elobjeto, el modo, elins tru men toy porúl timola finalidad. Tal y como se ha señalado, todos estos elementos que el autor extrae del "mótodbde caso' ten drán cumplida contes ta ción porme diodelosdes cripto res temáticos, el tipo de elementos de extracción de contenido utilizados en los servicios de gestión y el controldematerialaudiovisual.

Se ha de repetir la dualidad tan to en la na tu ra le za como en el origen de la in for ma ciónau diovisual televisiva, loquehacenecesa riocon tarconelementos paraladescripción de contenido tan to dela ban daimagen comodelaban da so nido, conlo cualsepotencialarecuperaciónsincronizadacomoasíncronaconrecursospotencialesytotales.

\section{Cuándo}

Jun to con elelemento analizadoconposterio ridad, elcuándono es unainterrogaciónque seobservecla ramenterefleja da en la formula ción deLass well, sin em bargo parece oportunocon tarconuna se rie de campos que con trolen y norma licen elaccesoalainformaciónpormediodedichoselementos.

Los datos cronológicos deben señalar tanto datos concretos como fechas entre las que puede estar comprendida la in forma ción. Los locativostemparales ${ }^{\circ}$ deben asemejar tanto los datos cronológicos de la información como de la captación de las imágenes, las cuales no siempre tienen porqué coin ci dir. En la ma yor parte delos hechos no ti cio sos, a ex cep ción de los di rec tos, real men te no se cuen ta con la in for ma ción en sí misma sino con las reacciones al acontecimiento acaecido con anterioridad,porloquepareceoportunocontarconelantecedentecronológico.

Porcuestiones eminentemente de control, la to talidad delas ba ses de da tos cuentan con la respuesta a otras preguntas que en nada ayu dan a contes taral pa radigma planteado como metodología del trabajo, pero que también pueden identificar dichas fe chas como son los da tos de pro duc ción y de emi sión del pro duc to in for ma tivoaudiovisual.

\section{Dónde}

Laidentificacióndelosluga resgeográ ficosqueseidentificanenelhechocomuni ca tivotam bién debe tener su si tio en el mar codelas ba ses de da tos docu men tales de

19 Ibidem

20 Ibidem 
las cadenas televisivas. Para ello los locativosidentificados describen diferentes aspectosreseñadosenlapiezainformativaaudiovisual.

Dichoslocativossepresentanen dife rentes camposparapodercontrolarlatotalidad de la tipología de posibles lugares geográficos en el documento audiovisual. Tambiénhaylocativos delugares físicosylocativosdelugardemarcado,ende finitiva tan to los lu ga res don de ocu rren los acon te ci mien tos como aque llos en los que concretamente serealiza la ac ción. Sede benobtenerloslugares referencia dos yloslugares vistos. Respecto a los lugares referenciados és tos se tra du ci rán en los te rri torios dela no ticia, mien tras quelos vis tos se rán tan to de si tios con cre tos como el es pe cíficoden trodeés tos, einclu so sede talla rálaiden tificacióna niveldecalles, edificios, etcéte ra.Los cam posconlos quese co rres pon de rían los señala dosen elpun to an te rior son:lugares deacción, pla nolugar,planolocaliza cióneimplicacióngeográfica. Cabe destacar en este caso el último campo por su peculiaridad y separación del método comunicativo de Lasswell ya que ofrece información referencial, independientemen te de quien fue vis to o es cu chó, y da tos rela cio na dos con países, es tados o zo nas geográficas (conjuntodepaísescondenominaciónarbitrada)implicadosenelhecho informativo.

\section{Contestación múltiple (qué, a quién = emisor / receptor; qué = mensaje; efectos) \\ Título facticio}

Sehapreferidodes tacartres campos quecontes tancues tiones diversas delmétodocomunicativodeLass well, derivadosen granmedidade suconfecciónentex tolibre, untipodelenguajequelejos deencorsetarellenguajedocumentalaportaflexibilidad para describir un tipo de texto aún poco estudiado, analizado y estandarizado parasuanálisisexhaustivoyconcluyente.

Elprime ro de es tos cam pos de con fec ción en tex to libre es el títu lo facticio, también denominado documental, en el cual el analista/documentalista ofrece en una fra seex plica tiva elemen tos cla ramenterefleja dos porLass well. Elquéy el quiénpa recen ine vi ta bles a la hora dela con fec ción de di cho título, que, aun que cuen ta con una confección sin normalizar, sí tiene elementos que lo normalizan para ayudar en el proceso de recupera ción docu mental, como pu die ran serlos tiemposverbales, elgéneroyelnúmero.

Ejemplodeestostítulossería:

\section{GeargeBush}

Ordena $\&$ ataque SobreIrak
GeargeBushordena $\mathrm{e}$ ataquesdoreIrak.

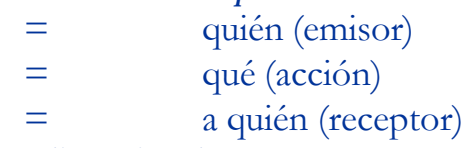

Estoselementoscognitivos pudieranimplementarseconotrosaspectossiempre ycuan dola lon gi tud del cam po no se ex ten die ra en ex ceso, y po drían am pliar secon otros elementos, comoluga resconcretos (dánde), fechas (aránd), etcétera. 


\section{Resumen}

Campotambién con fec cio na do en len gua jena tu ral que cuen ta con la particula ri daddeserde ma yorlongitud en nú me ro decaracteres, porloquelain forma cióncontenida en élre fleja, o pu die ra re flejar, to dos y cada uno de los ele men tos que con formandichomodelocomunicativo. Aho rabien, debequedarcla roquelasnormativas para la con fec ción de re sú menes de ar tícu los cien tíficos (tan to UNE como ISO) no son fórmulas válidas paraponerenefec toestaherramien ta documentalenlos de partamen tos de docu menta ción de las tele visio nes, lo que se de riva de la na tu ra le za audiovisual de los documentos y del carácter social, universaly enciclo pédico delainformacióncontenidaenlaspiezastelevisivas delosprogramasinformativos.

\section{Análisis cronológico}

Se ha re serva do para des cribir enúl ti molugar el cam pomás impor tan te delas bases de da tos do cumen tales de las empre sas au diovisuales, elcampo queen de finitiva es elquelo iden tifica ylo in dividualiza res pec to delos de más. El campo análisis cronológico,tambiénllamadocontenidoominutadoentreotrasposiblesdenominaciones, debe con tar con una corres pon den cia con el res to de cam pos de la base de da tos; es de cir, cual quier in for ma ción que esté plas ma da en este cam po de tex to libre de beráseñalarseenloscamposcorrespondientesdelenguajecontrolado.

Estacorrespondenciaseñaladaconanterio ridadhacequeseresuelvanlatotalidad delascues tiones plantea das has ta ahora, en de finitivaeste cam poes un fiel reflejo de to dos y cada uno delos ele men tos cog nos cen tes que se han exa mina do has ta aho ra. Tanto el quécontestado por descriptores o pun tos de acce so ono más ticos, comola acción descrita por los elementos temáticos, así como los locativos o los aspectos geográ ficos son narra dos en este campo; su utilidad es eviden te. Cualquierin formación que se encuentre en el campo de los elementos registrados debe con tar con su partefísicavistaoreferenciadaenelcampodelanálisiscronológico.

Este cam po es en de finitiva la des crip ción en tex to li bre delain for ma ción vi sual reflejadaysonorareferenciadaeneldocumentoobje todeanálisis, conformeambas sevansucediendoalolargodeltiempo.

Son va rios los ele men tos que con forman este cam po, en pri merlugary como método de ubicación de las imágenes en el soporte se indican los datos cronológicos, hora, minuto y segundo del comienzo del material. Por su naturaleza televisiva, de origen inicialmente cinematográfico, se alude a la información referencial, a la incidenciaangularyalosmovimientosdecámarayencuadre.

Como último elemento se describe en texto libre el desarrollo de la acción y los efec tos deésta, para ello se ten drán que iden tifi car se gún el acon te ci mien to, ele mentos como el qué aándb dóndegenérico, dóndeespecífico, parqué quiénla rea li za, quiénla re cibe, et cé te ra. Porlo tan to di cho cam po con tes ta a cada una delas cues tio nes planteadasporlafórmuladecomunicacióndeLasswell. 


\section{Investigadón Biblidtedóógicav. 18 N o. 37 julio/ diciembre de 2004}

\section{0:00:05 PMGaspar Llamazares, coondinadongeneral delzquierdaUnida, señalaennuela deprensaenlasedemadileñalainconguendiadlgobiernodeA znarpordapoyoincondicianalala políticagdpistadeGeargeBushenIrakyendmunda}

Sise analizalain formación señala da porla des crip ción de estetotal, ${ }^{21}$ se po drá observar cómo se contesta a diferentes elementos de la fórmula de comunicación. Como el quiénrea liza la ac ción(Gas par Lla ma za res), y en este caso aquiérepresenta oen nombredequé orga niza ción (IzquierdaUnida), ein di casobrequiénestádirigi dala ac ción(George Bush) y so bre qué lugaresgenéricos repercu tela ac ción(Iraky elmundo), asícomo ello ca tivo es pe cífico cóndeocu rrela noticia (sede de Iz quier da Unida enMadrid); además de esto elquésein dicarápormediodelosdes crip to res temáticos que aludirían a pdíticaintemadional, al apgoincondicional, a los conflidosamados y tambiénincluiría otrosdescriptoresconlosquecon taralapropiaherramienta.

Porlo tan to, y como pue deob servarse, este cam poesimpres cin dibleparalalo calización de las imágenes en el soporte además de la aprehensión e identificación de los diferentes iconos que conforman la pieza de información, conformación que puedeadherirsedeformasincronizadasiseatienealaimagenyalsonido.

\section{CONCLUSIONES}

No se de sea ha cerun re su mengeneral del ar tícu lo en esteapar ta do de con clu siones, sinembargo pareceadecuadodesta carunaseriedeaspectosque pueden parecer oportunos y relevantes para todo aquel que con posterioridad pudiera utilizar este métodoparadesarrollarbases dedatosdocumentales.

En primer lugar este método es especialmente válido para bases de datos documentalesperiodísticasynoparalaimplementacióndeotrasherramientasdocumentales en instituciones documentales donde el modelo comunicativo sea diferente; ejemplosdeellosonlosqueanalizanyges tionanámbitoscientíficos.

Sehatratadodedemostrarcómo contestaunaherramientadocumentalplanteada en un trabajo previo a las consultas planteadas por Lasswell y cómo, al igual que el mensaje perio dís tico, se con for ma con es tos ele men tos la base de da tos, al descomponerlosparacon poste rio ridad consul taryaccederalain formación porlasmismas cuestionesplanteadasporlosperiodistas.

Esteméto do provo ca unaade cuaciónen trelos men sajes en via dos porelemisorperiodista-cadenaysuconservacióny recuperaciónenlabasede datos. Adecuación que hace posible que la realidaddocumental sea absolutamentecercanaalquehacer

21 Periodísticamentesedis tinguenlasimágenesentrerecursosy to tales. Éstos corresponderíancon las de cla raciones delos personajes, mien tras quelos re cursos se rían ac ciones. En elperiodismoactuallas de cla ra cio nes son una ma ne ra muy co mún de ac ce dera la in for ma ción, tan to esasí que una gran parte delain for ma ción es di fun dida pores tos me dios, se ñalan do asíel me dio que la in for ma ción es veraz desde el momento que surge y es comentada por la propia fuente. 
co tidiano delos comunicadores. Nodebeolvidarsequelosges tores dein formación es tánalservicio de sus usua rios, de aquellos bene fi cia rios rea les delos pro ductos yde los serviciosdocumentales, porloque todo el proce so de análisis yges tión, todalacadenadocumental, y en definitiva todo el diseñoyorganización debenestarencaminados a dar buen cum plimien to al obje tivoprin ci paldela uni dad docu men tal: ofrecerserviciosalosusuarios deformaeficien teyrápida.

\section{BiBLIOGRAFÍA}

ABADALFALGUERAS, E. “Diseñoycreacóndaunabasedadatosenunmediode comnicaión", en: Manual deDoumentacoón Peiodística Madrid: Síntesis, 1995, pp. 195-211.

AGIRREAZALDEGI BERRIOZABAL, Teresa.Elusodeladbaimentacoónalt dioisual en los programasinfomativos diarios detdeisión [Bilbao]: Servicio de Publicaciones de la Universidad del País Vasco, 1996.

AGUADO, Fran cis coJa vier. Organiza ción del sis te ma de archivoy do cur men ta ción de Pren sa Es pañola ( $A B C$ Blan co y Ne gro), en RevistaGene ral deInfomadónyDœumetadón 5, núm. 2, 1995, p. 203-208.

AMARO, Regina Keiko O.F. "Analise do discurso: textos básicos para a análisedocumentaria”, en Analisedbamentania: consideraçốsteéricasexpe nimentaçôes Sâo Paulo: FESAB, 1989, p. 59 - 70.

AMAT,CarlosBenito. "Facto res condicionantes delas operacionesdocur mentales en medios de comunicación", en RevistaEspañoladeDoamen tacónCientífica, 19, núm. 2, 1996, p.188-201.

AROZIN LÓPEZ, Virtudes; Lopez Bruñet, Trinidad. "Problemas que presen tala des crip ción de imáge nes enla elabo ra ción de bases de da tos multimedia", en Cuartas Jomadas Españdas de Doamentación Científica Gijón: Universidad de Oviedo. Servicio de Publicaciones, 1994, p. 411-416.

CACHO, Luis F.Archivosaudioisuales Cinevideo, $\mathrm{n}^{\circ}$ 153, sep tiem bre 1998, p. 25-32.

CEBRIAN HERREROS, Mariano. Infomadón audioisual. Coneqdo, ténica, expreiónyaplicadones Madrid: Síntesis, 1995.

CEBRIAN HERREROS, Mariano. Infomadóntdeisiva: mediaciones, contenidos, expreiónyprogamaión Madrid: Síntesis, 1998.

COMISIÓNDE TÉCNICOSDEARCHIVO SONOROYDOCUMENTACIÓN. NormasdacatalogacónddanchivosonorodeRadioNacionaldeEspaña. Madrid: Instituto Oficial de Radio y Televisión, 1992. 2 vol.

CONESA, Alicia. "La do cu men ta ción en los me dios de co mu nica ción aut diovisual", en Manual de Doumentadón Peiodística Madrid: Síntesis, 1995, p. 147-159. 
CONESA, Ali cia. "Lesenissionsderàdioytelevisò̀ unpatimoniaconservar", en LaDcametadóals Mitjand d'informaió L'experènia Multimèda València: Generalitat Valencia, 1994, p. 283-290.

CORRAL BACIERO, Manual.Lasbasesdedatosene periodismoaudiovisual: $\mathrm{A}$ tiempoes d tirano Mensajes y Medios, agosto-septiembre 1989, $\mathrm{n}^{\circ}$ 6, p. 76-80.

DOCUMENTACIÓalsMitjansd'informadóL 'experiendiamiltimødia.Ponénces ycondusions, la(Actas). Valen cia, Palau de Pine da, 7-9 marzo. Valen cia: Generalitat Valenciana, 1994.

DOCUMENTS that moveand speak: audioisual archives in thenewinfomation age proceedingsofasymposiummganizeelforthelnternational CandiloArdives bytheNational Ardives of Canada München: Saur, 1992.

ECO, Um ber to [et. al.]. Primapropostaperunmoddlodincercainterdisaiplinare sulrapportotelevisione/ pubdico Perugia:Ins ti tu to diEtnología e Antropología Culturale, 1965.

ECO, Umberto; Fabri, P. "Progetto di ricerca sull'utilizzaziones dell' informaziones ambiental", en Prodlemi ddl 'infomrziones 1978, n 4, pp. $555-597$.

FOURNIAL, Catherine. “Análisis documentaldeimágenes enmovimiento", en Panoramaddosanchivosaudiovisuales Ma drid:Servicio de Publicaciones del Ente Público RTVE, 1986. p. 249-258.

GALDÓN LÓPEZ, Gabriel. Pinäpiosqperativosdedbamentadónperiodísica. Madrid: Dossat, 1989.

GALDÓN LÓPEZ, Gabriel. "Principios configuradores de la actividad documental periodística", en Dœumtatacón delas Cienias dela Infomadớn v. XI, 1985, pp. 249-265.

García GutiérReZ, Antonio. Procedimientos deanálisis dbumental automática EstudiodacasaSe villa:Ins ti tu to An daluz delPa trimonio His tórico, 1996

GONZÁLEZ REQUENA,Je sús.Eldisarsotelevisiva espetáaulodlapostmø demidad Madrid: Cátedra, 1988.

HARRISON, Ha rriet W. Who, what, whenandwhy?A ccesstofilmsthoughthecat taloge IFLA Journal, Vol. 18 (3), 1992, p. 238-242.

HERNÁNDEZ PÉREZ, An to nio. Doamentadónaudiovisual:mtodbloǵapara d análisis dboumental dela infommaón periodística audiovisual. Madrid: Universidad Complutense, 1992.

HIDALGO GOYANES, Paloma. Análisis documental de audiovisuales, en Introdurción a la dbammitacón infomativa y period'sitca Sevilla: MAD, 1999, pp. 333-350. 
LÓPEZ DE QUINTANA, Eugenio. "Documentación en televisión", en Manual dedoumentacón informativa. Madrid: Cátedra, 2000, p. 83-181.

LÓ PEZ PUMAREJO, Tomás Alberto. "El discurso televisual: modelos culturales y vida cotidiana”, en Eutopias Vol. 2, n.1 (invierno 1986) p. 161-171.

LÓPEZ YEPES, Alfonso. Manual dedoumentadón audiovisual. Pamplona: Universidad de Navarra, 1992.

LOZANO, Jorge;Peña-Marin, Cris tina;Abril, Gonzalo. Análisisdiddisar sa hada una semiótica delainteracción textual. Madrid: Cátedra, 1989.

MANUALdedbamentacióaudiovisualenràdioitelevisióValèn cia:Universitat, 1999.

MANUAL dedoumentacón infomativa Madrid: Cátedra, 2000.

MANUAL dedoumentadón periolística Madrid: Síntesis, 1995.

MCLUHAN, Marshall. LagalaxiaGutenbeggénesisdd "homo typographicus". Barcelona: Círculo de Lectores, 1993

NORRLANDER, Stellan. “The mi ni mun le gal data list", en Panoramadelos ardivosaudiovisuales Madrid:Serviciode Publica ciones delEntePúblico RTVE, 1986, p. 239.248.

O'CONNOR, Brian. "Access to moving image documents: background concepts and proposals for surrogates for film and video works", en TheJarnal ofDoamentation Vol.41, n. 4, (de cem ber 1985), p. 209- 221.

OWEN, C. Mkadata for musicandmovies Audiovisual Librarian, 24, 1, p. 47-50.

RODES, Jean-Michel. "Analyse et indexation par 1 ímage, les voies d'une automatisation", en Dossiesdel'Audovisud, 1992, n 45 núme ro es pe cial, p. 77-78.

SANABRIA, Fran cis co.Informacónaudiovisual:tenáayténicadlainformación radiofónica ytdevisiva Barcelona: Bosch Comunicación, 1994.

SHANNON, Claude E. Tenáa matemática dela commicacón Madrid: Forja, 1981.

WOLF, Mau ro.Lainves tiga ción dela co munica ción demasas. Barcelona: Paidós, 1991.

WRIGHT MILLS, C. PonerpoliticandpeopleNew York: Ox ford Uni ver sity Press, 1963

ZUÑIGA, Joseba. ComnicadónAudioisual. San Sebastián: Escivi, 1998.
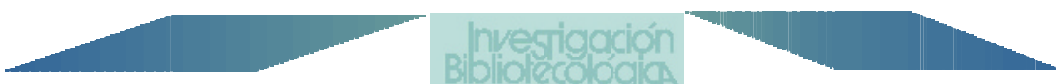